\title{
Cervical Sagittal Alignment Parameters of Patients Admitted to Neurosurgery and Emergency Clinics in a State Hospital at Eastern Part of Turkey
}

\author{
Sümeyye Çoruh Kaplan', Murat Şakir Ekşi \\ ${ }^{I}$ Department of Neurosurgery, Diyarbakir Bismil State Hospital, Diyarbakir, Turkey \\ ${ }^{2}$ Department of Orthopedic Surgery-Spine Center, University of California at San Francisco, California, USA
}

\begin{abstract}
Objective: Cervical spine encompasses a bridge role between the head and the lower parts of the spine and therefore has unique properties. Our aim in this study was to evaluate the cervical sagittal alignment parameters in pediatric and adult nonsurgical patients and to find any differences in respect of age, sex and admission type.

Methods: All patients who were admitted to emergency and neurosurgery clinics of Diyarbakir Bismil State Hospital due to cervical spine problems (trauma, radiculopathy, paraspinal pain) in 2014 were enrolled retrospectively into the study. Cervical anteriorposterior and lateral X-rays were obtained. Our exclusion criteria were cervical coronal deformity, multitrauma, Glasgow Coma Scale $<15$, traumatic disruption of the cervical spine, history of malignancy, spinal infection, metabolic or rheumatologic diseases. Results: There were 44 female and 55 male patients $(n=99)$ in the study. Thirty-five $(35.35 \%)$ of the patients were younger than 18 years of age. Mean cervical spinal alignment parameters were as follows: $-42.81 \pm 11.23^{\circ}(\mathrm{OC} 2),-17.15 \pm 11.48^{\circ}(\mathrm{C} 2-\mathrm{C} 7)$, $-29.82 \pm 7.60^{\circ}$ ( $\mathrm{T} 1$ slope), $-3.62 \pm 3.05^{\circ}$ (C3), $-3.14 \pm 3.05(\mathrm{C} 4),-3.80 \pm 2.74^{\circ}$ (C5), $-3.12 \pm 2.36^{\circ}$ (C6), $-3.43 \pm 2.53^{\circ}$ (C7). Positive correlations were observed between age-C2C7 angle, $\mathrm{C} 2 \mathrm{C} 7$ angle-T1 slope, $\mathrm{C} 3$ angle-C4 angle, $\mathrm{C} 4$ angle-OC2 angle, $\mathrm{C} 4$ angle-T1 slope, $\mathrm{C} 4$ angle- $\mathrm{C} 5$ angle. The one only negative correlation was between $\mathrm{OC} 2$ angle- $\mathrm{C} 2 \mathrm{C} 7$ angle.

Conclusion: In this regional study, it has been observed that global cervical lordosis increases as age increases. C4 vertebra is in the middle of this evaluation as it has many correlations with other cervical segments, which should be kept in mind when making surgical plans for this delicate spine region.
\end{abstract}

Key Words: Sagittal alignment $\cdot$ Cervical spine $\cdot$ Radiography $\cdot$ Neurosurgery $\cdot$ Emergency

\section{INTRODUCTION}

Spinal alignment has been the area of interest with a more comprehensive understanding of the underlying mechanisms. Dubousset described the spinal balance in the economic range

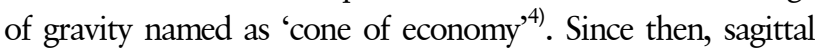
and coronal parameters of thoracolumbar spine with pelvic parameters have been thoroughly studied ${ }^{3,9,11)}$. Cervical spine encompasses a bridge role between the head and the lower parts of the spine and therefore has unique properties; however

- Received: March 5, 2015 - Revised: June 5, 2015

- Accepted: June 15, 2015

Corresponding Author: Murat Şakir Ekşi, MD

Department of Orthopedic Surgery, University of California at San

Francisco, 500 Parnassus Avenue MU320 West, San Francisco, CA

94143-0728, USA

Tel: + 1-415-4761167, Fax: + 1-415-4761304

E-mail: muratsakireksi@gmail.com/Murat.Eksi@ucsf.edu

*The authors contributed equally to the study.

$\otimes$ This is an Open Access article distributed under the terms of the Creative

Commons Attribution Non-Commercial License (http://creativecommons.org/ licenses/by-nc/3.0/) which permits unrestricted non-commercial use, distribution, and reproduction in any medium, provided the original work is properly cited. cervical spinal alignment has not been studied as much as thoracolumbar spinal alignment. The studies are scarce even in the pediatric population ${ }^{1)}$.

Our aim in this study was to evaluate the cervical sagittal alignment parameters in pediatric and adult non-surgical patients and to find any differences in respect or age, sex and admission type. We analyzed cervical lateral X-rays of 99 patients that admitted to a state hospital's emergency and neurosurgery clinics at eastern part of Turkey.

\section{MATERIALS AND METHODS}

All patients who were admitted to emergency and neurosurgery clinics of Diyarbakir Bismil State Hospital due to cervical spine problems (trauma, radiculopathy, paraspinal pain) in 2014 were enrolled retrospectively into the study. The study protocol was compatible with 1964 Declaration of Helsinki. Cervical anterior-posterior (A-P) and lateral X-rays (head to T2) were obtained while the patients stood in a relaxed position and hands were flexed over clavicles in adults and teenagers. The cervical x-ray of the younger ones was obtained with the 
assistance of one of the parents. The patients were asked to look straightforward during the procedure. Cervical A-P films were evaluated only for presence of coronal deformity (Cobb $>10^{\circ}$ ). Our exclusion criteria were cervical coronal deformity, multitrauma, Glasgow Coma Scale $<15$, traumatic disruption of the cervical spine, history of malignancy, spinal infection, metabolic or rheumatologic diseases. Pediatric age limit was selected as 18 years. After exclusion criteria, 99 patients were found to be suitable for the study.

Lateral cervical spinal $\mathrm{x}$-rays were assessed in respect of occipito-C2 angle (OC2) (between MacGregor line and inferior end-plate of $\mathrm{C} 2$ vertebral corpus), C2-C7 angle (between inferior end-plates of C2 and C7), T1 slope (between horizontal line and upper end-plate of $\mathrm{T} 1$ vertebral corpus), and segmental vertebral angles from $\mathrm{C} 2$ to $\mathrm{C} 7$ (between upper and lower end-plates of each corresponding vertebral corpus) (Fig. 1). For accuracy of the data, all measurements were done by one of the authors (S.C.K.) on a software program (Surgimap, Nemaris Inc, New York, USA).

Dichotomous and continuous variables were evaluated with chi-square and student's t-test, respectively. Correlation study was done with Pearson's correlation. All statistical analyses were conducted with SPSS 22.0 (Statistical Package for the Social Sciences, IBM, New York, USA). An alpha value of
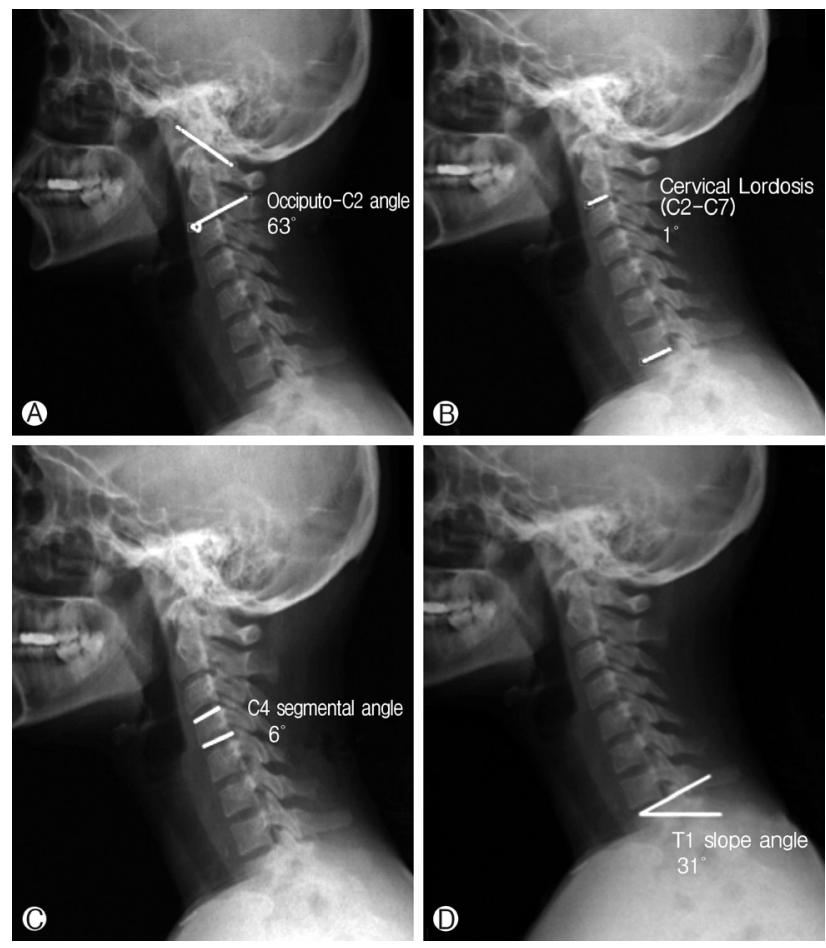

Fig. 1. Cervical sagittal alignment parameters: (A) Occiputo-C2 angle, (B) C2-C7 angle (cervical lordosis), (C) C4 segmental angle, (D) T1 slope.
$<0.05$ was accepted as significance of difference.

\section{RESULTS}

There were 44 female and 55 male patients in the study. Mean age of the cohort was $30.30 \pm 18.90$ years. Thirty-five (35.35\%) of the patients were younger than 18 years of age. Mean cervical sagittal alignment parameters were as follows: $-42.81 \pm 11.23^{\circ}$ (OC2), $-17.15 \pm 11.48^{\circ}$ (C2-C7), $-29.82 \pm 7.60^{\circ}$ (T1 slope), $-3.62 \pm 3.05^{\circ}(\mathrm{C} 3),-3.14 \pm 3.05$ (C4), $-3.80 \pm 2.74^{\circ}$ (C5), $-3.12 \pm 2.36^{\circ}$ (C6), $-3.43 \pm 2.53^{\circ}$ (C7). Patients were compared according to clinical admission type, gender and their pediatric or adult status (Table 1-3). Statistical difference was present in age $(p=0.007)$ and $C 4$ angle $(p=0.003)$ between patients admitted to emergency clinic and patients admitted to neurosurgery clinic. All other cervical sagittal alignment parameters were similar between the groups. More female patients were admitted to emergency department $(61.4 \%$ vs. $40.0 \%$, $\mathrm{p}=0.044)$ (Table 4).

Table 1. Comparison of cervical sagittal alignment parameters according to patient gender

\begin{tabular}{lccl}
\hline \hline & Male $(\mathrm{n}=55)$ & Female $(\mathrm{n}=44)$ & $\mathrm{p}$-value \\
\hline Age & $28.4 \pm 18.16$ & $32.68 \pm 19.75$ & 0.265 \\
Occiput-C2 $\left(^{\circ}\right)$ & $-41.03 \pm 9.27$ & $-45.04 \pm 13.06$ & 0.08 \\
C3 angle $\left(^{\circ}\right)$ & $-3.72 \pm 3.24$ & $-3.5 \pm 2.84$ & 0.715 \\
C4 angle $\left(^{\circ}\right)$ & $-2.76 \pm 2.72$ & $-3.61 \pm 3.39$ & 0.170 \\
C5 angle $\left(^{\circ}\right)$ & $-3.72 \pm 2.81$ & $-3.90 \pm 2.68$ & 0.745 \\
C6 angle $\left(^{\circ}\right)$ & $-3.4 \pm 2.25$ & $-2.77 \pm 2.46$ & 0.190 \\
C7 angle $\left(^{\circ}\right)$ & $-3.63 \pm 2.34$ & $-3.18 \pm 2.76$ & 0.378 \\
T1 slope $\left({ }^{\circ}\right)$ & $-29.41 \pm 8.08$ & $-30.34 \pm 7.01$ & 0.551 \\
C2-C7 angle $\left({ }^{\circ}\right)$ & $-16.32 \pm 10.16$ & $-18.18 \pm 13.00$ & 0.427 \\
\hline
\end{tabular}

Table 2. Comparison of cervical sagittal alignment parameters according to patients' admission to neurosurgery or emergency clinic

\begin{tabular}{llcl}
\hline \hline & $\begin{array}{c}\text { Neurosurgery } \\
\text { Clinic }(n=50)\end{array}$ & $\begin{array}{c}\text { Emergency } \\
\text { Clinic }(n=49)\end{array}$ & $p$-value \\
\hline Age & $35.28 \pm 20.17$ & $25.22 \pm 16.19$ & 0.007 \\
Occiput-C2 $\left(^{\circ}\right)$ & $-41.4 \pm 10.66$ & $-44.26 \pm 11.72$ & 0.206 \\
C3 angle $\left(^{(}\right)$ & $-3.06 \pm 2.75$ & $-4.20 \pm 3.25$ & 0.062 \\
C4 angle $\left(^{\circ}\right)$ & $-2.24 \pm 2.04$ & $-4.06 \pm 3.61$ & 0.003 \\
C5 angle $\left(^{\circ}\right)$ & $-3.64 \pm 2.28$ & $-3.97 \pm 3.16$ & 0.543 \\
C6 angle $\left(^{\circ}\right)$ & $-2.74 \pm 2.04$ & $-3.51 \pm 2.60$ & 0.105 \\
C7 angle $\left(^{\circ}\right)$ & $-2.35 \pm 2.35$ & $-3.40 \pm 2.73$ & 0.92 \\
T1 slope $\left(^{\circ}\right)$ & $-28.68 \pm 8.03$ & $-31 \pm 7.02$ & 0.13 \\
C2-C7 angle $\left(^{\circ}\right)$ & $-19.38 \pm 12.67$ & $-14.87 \pm 9.74$ & 0.051 \\
\hline
\end{tabular}


Table 3. Comparison of cervical sagittal alignment parameters between pediatric and adult patients

\begin{tabular}{lccc}
\hline \hline & $\begin{array}{c}\text { Pediatric } \\
(\mathrm{n}=35)\end{array}$ & $\begin{array}{c}\text { Adult } \\
(\mathrm{n}=64)\end{array}$ & p-value \\
\hline Occiput-C2 $\left(^{\circ}\right)$ & $-42.22 \pm 10.67$ & $-43.14 \pm 11.60$ & 0.701 \\
C3 angle $\left(^{\circ}\right)$ & $-3.40 \pm 2.77$ & $-3.75 \pm 3.21$ & 0.588 \\
C4 angle $\left({ }^{\circ}\right)$ & $-3.17 \pm 3.59$ & $-3.125 \pm 2.74$ & 0.942 \\
C5 angle $\left({ }^{\circ}\right)$ & $-3.45 \pm 2.97$ & $-4.00 \pm 2.61$ & 0.349 \\
C6 angle $\left(\left(^{\circ}\right)\right.$ & $-2.94 \pm 2.20$ & $-3.21 \pm 2.45$ & 0.581 \\
C7 angle $\left(^{\circ}\right)$ & $-3.54 \pm 2.51$ & $-3.37 \pm 2.56$ & 0.754 \\
T1 slope $\left(^{\circ}\right)$ & $-30.02 \pm 7.56$ & $-29.71 \pm 7.68$ & 0.847 \\
C2-C7 angle $\left({ }^{\circ}\right)$ & $-15.37 \pm 9.11$ & $-18.12 \pm 12.55$ & 0.256 \\
\hline
\end{tabular}

Table 4. Clinical admission type according to gender and age

\begin{tabular}{lllc}
\hline \hline & Neurosurgery & Emergency & $\mathrm{p}$-value \\
\hline Female & $17(38.6 \%)$ & $27(61.4 \%)$ & 0.044 \\
Male & $33(60 \%)$ & $22(40 \%)$ & \\
Pediatric & $13(37.1 \%)$ & $22(62.9 \%)$ & 0.06 \\
Adult & $37(57.8 \%)$ & $27(42.2 \%)$ & \\
\hline
\end{tabular}

Table 5. Significant correlations between demographic data and cervical sagittal parameters

\begin{tabular}{lcc}
\hline \hline & $\mathrm{R}$ value & $\mathrm{p}$-value \\
\hline Age-C2C7 angle & 0.264 & 0.008 \\
$\mathrm{C} 2 \mathrm{C} 7$ angle-T1 slope & 0.361 & 0.000 \\
$\mathrm{C} 3$ angle-C4 angle & 0.245 & 0.014 \\
$\mathrm{C} 4$ angle-OC2 angle & 0.276 & 0.006 \\
C4 angle-T1 slope & 0.207 & 0.04 \\
C4 angle-C5 angle & 0.254 & 0.011 \\
OC2 angle-C2C7 angle & -0.248 & 0.013 \\
\hline
\end{tabular}

Positive correlations were observed between age-C2C7 angle $(\mathrm{R}=0.264, \mathrm{p}=0.008), \mathrm{C} 2 \mathrm{C} 7$ angle- $\mathrm{T} 1$ slope $(\mathrm{R}=0.361$, $\mathrm{p}=0.000), \mathrm{C} 3$ angle- $\mathrm{C} 4$ angle $(\mathrm{R}=0.245, \mathrm{p}=0.014), \mathrm{C} 4$ angleOC2 angle $(\mathrm{R}=0.276, \mathrm{p}=0.006), \mathrm{C} 4$ angle-T1 slope $(\mathrm{R}=0.207$, $\mathrm{p}=0.04)$, $\mathrm{C} 4$ angle- $\mathrm{C} 5$ angle $(\mathrm{R}=0.254, \mathrm{p}=0.011)$. The one only negative correlation was between $\mathrm{OC} 2$ angle-C2C7 angle $(\mathrm{R}=-0.248, \mathrm{p}=0.013)($ Table 5).

\section{DISCUSSION}

Cervical spine is unique in respect of its wide range of motion capability and carrying the head over it. Cervical sagittal alignment is important not only for stability of motion in the 'cone of economy', but also preventing cervical spinal cord myelopathy $^{2)}$. It was demonstrated that spinal cord intramedullary pressure increased with increased amount of kyphosis, ${ }^{5,14)}$. The effect of changes is not limited to only morphological cord changes, but also influences quality of life of the patients significantly ${ }^{2}$. Morphometric radiographical studies dedicated to cervical sagittal alignment are rare in the literature.

There are several measurement methods that could be conducted to assess cervical sagittal alignment. Although Harrison posterior tangent method is superior over Cobb method in measuring cervical lordosis, it has been shown that good intra- and inter-rater reliability of Cobb method and its clinical use are easier than Harrison method ${ }^{8,13,15)}$. Cervical lordosis measurement with $\mathrm{C} 1-\mathrm{C} 7$ or $\mathrm{C} 2-\mathrm{C} 7$ has been performed and has been found that they have been overestimating and underestimating cervical lordosis, respectively ${ }^{2}$. We preferred C2-C7 sagittal Cobb angle to measure cervical lordosis of the patients. We also used occiput to $\mathrm{C} 2$ angle to involve all cervical spinal segments into consideration. In our final analysis, OC2 angle was found to be significantly negatively correlated with global cervical lordosis (C2-C7 angle). This makes sense as global cervical lordosis increases, OC2 angle decreases to keep the head in horizontal plane. Cervical lordosis angle of our cohort was $17.15 \pm 11.48^{\circ}$ which is comparable to age-paired studies in the literature ${ }^{6}$. Increased age was found to be correlated with increased cervical lordosis, which is also comparable to current literature ${ }^{6,7}$. This could be explained in part by compensatory alignment changes in cervical spine due to whole spine degenerative process ${ }^{12}$. However, it may not be valid for segmental measurements. When we compared cervical parameters of the patients admitted to neurosurgery and emergency clinics, we saw that all cervical parameters except global cervical lordosis (C2-C7) were higher in patients admitted to emergency clinic even though they were younger than the patients admitted to neurosurgery clinic. Global cervical lordosis was still higher in patients admitted to neurosurgery clinic. Segmental vertebral angles ( $\mathrm{C} 3$ to $\mathrm{C} 7$ ) were higher in our study. This can be due to the differences in the study protocols. As a shortcoming of this study, patients who applied with cervical trauma and/or cervical pain signs and symptoms to our clinics were enrolled into the study. Even though severe cases and obvious pathologies were excluded from the study protocol, we compared our results with the asymptomatic case-series presented in the literature.

We observed that many correlations in the cervical sagittal alignment were involving $\mathrm{C} 4$ vertebral corpus (with OC2, C3, C5, T1 slope). This may depict the importance of C4 vertebral corpus position in the cervical spine, which should be considered in surgical treatment planning of patients. There was a significant difference in $\mathrm{C} 4$ segmental angle between patients admitted to neurosurgery and emergency clinics. To make a more comprehensible statement, prospective studies with large cohorts of samples representing general population are in need.

T1 slope has gained increased awareness among spine sur- 
geons, recently. Studies about this angle are still limited in the literature. It was found that $\mathrm{T} 1$ slope has a significant correlation with cervical alignment and cervical lordosis ${ }^{2,10}$. We also observed a positive correlation of T1 slope with both global cervical lordosis and $\mathrm{C} 4$ segmental angle.

All spine segments are related with each other and when one of them changes, the others are affected in a contiguous way: increased pelvic incidence leads to increased lumbar lordosis, increased lumbar lordosis leads to increased thoracic kyphosis, increased thoracic kyphosis leads to increased cervical lordosis ${ }^{2)}$. Our study protocol included only cervical A-P and lateral X-rays, which were obtained for clinical evaluation of the relevant patients. So, we could not compare our results in cervical spine with other spinopelvic parameters. However, it is obvious that for a good restoration of cervical alignment in surgical patients, a whole 3-ft spine films from head to upper femoral shaft should be evaluated.

\section{CONCLUSION}

In this regional study, it has been observed that global cervical lordosis increases as age increases. C4 vertebra is in the middle of this evaluation as it has many correlations with other cervical segments, which should be kept in mind when making surgical plans for this delicate spine region. To make a more comprehensible statement about asymptomatic general population, more prospective morphometric studies should be made.

\section{ACKNOWLEDGEMENT}

Murat Şakir Ekşi, M.D. was supported by a grant from Tubitak (The Scientific and Technological Research Council of Turkey), Grant number: 1059B191400255.

\section{REFERENCES}

1. Abelin-Genevois K, Idjerouidene A, Roussouly P, Vital JM, Garin C: Cervical spine alignment in the pediatric population: a radiographic normative study of 150 asymptomatic patients. Eur Spine J 23:1442-1448, 2014

2. Ames CP, Blondel B, Scheer JK, Schwab FJ, Le Huec JC, Massicotte EM, et al: Cervical radiographical alignment: comprehen- sive assessment techniques and potential importance in cervical myelopathy. Spine (Phila Pa 1976) 38:S149-160, 2013

3. Ames CP, Smith JS, Scheer JK, Bess S, Bederman SS, Deviren $\mathrm{V}$, et al: Impact of spinopelvic alignment on decision making in deformity surgery in adults: A review. J Neurosurg Spine 16:547-564, 2012

4. Dubousset J: Three-dimensional analysis of the scoliosis deformity, Weinstein S (ed): The Pediatric Spine: Principles and Practice. New York: Raven pp. 479-496, 1994

5. Farley CW, Curt BA, Pettigrew DB, Holtz JR, Dollin N, Kuntz C 4th: Spinal cord intramedullary pressure in thoracic kyphotic deformity: a cadaveric study. Spine (Phila Pa 1976) 37:E224-230, 2012

6. Gore DR, Sepic SB, Gardner GM: Roentgenographic findings of the cervical spine in asymptomatic people. Spine (Phila Pa 1976) $11: 521-524,1986$

7. Hardacker JW, Shuford RF, Capicotto PN, Pryor PW: Radiographic standing cervical segmental alignment in adult volunteers without neck symptoms. Spine (Phila Pa 1976) 22:1472-1480, 1997

8. Harrison DE, Harrison DD, Cailliet R, Troyanovich SJ, Janik TJ, Holland B: Cobb method or Harrison posterior tangent method: which to choose for lateral cervical radiographic analysis. Spine (Phila Pa 1976) 25:2072-2078, 2000

9. Klineberg E, Schwab F, Smith JS, Gupta MC, Lafage V, Bess S: Sagittal spinal pelvic alignment. Neurosurg Clin N Am 24: 157-162, 2013

10. Knott PT, Mardjetko SM, Techy F: The use of the T1 sagittal angle in predicting overall sagittal balance of the spine. Spine J 10:994-998, 2010

11. Mac-Thiong JM, Labelle $H$, Roussouly P: Pediatric sagittal alignment. Eur Spine J 20 Suppl 5:586-590, 2011

12. Oh T, Scheer JK, Eastlack R, Smith JS, Lafage V, Protopsaltis TS, et al: Cervical compensatory alignment changes following correction of adult thoracic deformity: a multicenter experience in 57 patients with a 2-year follow-up. J Neurosurg Spine 22: 658-665, 2015

13. Polly DW, Jr., Kilkelly FX, McHale KA, Asplund LM, Mulligan M, Chang AS: Measurement of lumbar lordosis. Evaluation of intraobserver, interobserver, and technique variability. Spine (Phila Pa 1976) 21:1530-1535, 1996

14. Shimizu K, Nakamura M, Nishikawa Y, Hijikata S, Chiba K, Toyama Y: Spinal kyphosis causes demyelination and neuronal loss in the spinal cord: a new model of kyphotic deformity using juvenile Japanese small game fowls. Spine (Phila Pa 1976) 30: 2388-2392, 2005

15. Singer KP, Jones TJ, Breidahl PD: A comparison of radiographic and computer-assisted measurements of thoracic and thoracolumbar sagittal curvature. Skeletal Radiol 19:21-26, 1990 